\title{
Strongly Convergence Theorem of m-accretive Operators
}

\author{
Yujun Liu \& Sumei Xu \\ Department of Mathematics, Tianjin Polytechnic University, Tianjin 300160, China \\ Department of Mathematics and Applied Mathematics, Anyang Normal University, Anyang 455000, China \\ E-mail: liuyjun@yahoo.com.cn
}

\section{Abstract}

Let $X$ be a real Banach space, we will introduce a modifieations of the Mann iterations in a uniformly smooth Banach space, $x_{n+1}=\alpha_{n}\left(\lambda u+(1-\lambda) x_{n}\right)+\left(1-\alpha_{n}\right) J_{r} x_{n}$, Where $\left\{\alpha_{n}\right\}, \lambda$ satisfied some conditions, then we will prove the strongly convergence of the sequence $\left\{x_{n}\right\}$ to a zero of accretive operators. This theorem extend (Kim and Xu, Nonlinear Analysis) results. (Kim, 2005, pp.51-60)

Keywords: Strong convergence, Non-expansive mapping, Accretive operator

\section{Preliminaries}

Recall that a (possibly multivalued) operator A with domain $D(A)$ and $R(A)$ in $X$ is accretive if for each $x_{i} \in D(A)$, and $y_{i} \in A x_{i}(i=0,1,2)$, there exits a $j \in J\left(x_{2}-x_{1}\right)$ such that $\left(y_{2}-y_{1}, j\right) \geq 0$. An accretive operator $A$ is $\mathrm{m}$-accretive if $R(I+r A)=X$, for each $r>0$. The set of zero of $A$ is denoted by $F$. Hence $F=\{z \in D(A): 0 \in A(z)\}=A^{-1}(0)$. We denote by $J_{r}$ the resolvent of $A$. Note that if $A$ is $\mathrm{m}$-accretive then $J_{r}: X \rightarrow X$ is nonexpansive and $\mathrm{F}\left(J_{r}\right)=\mathrm{F}$.

In order to prove our results, we need the following lemmas:

Lemma 1: Assume $\left\{\alpha_{n}\right\}$ is a sequence of nonnegative numbers such that (Xu, 2002, pp.240-256)

$\alpha_{n+1} \leq\left(1-\gamma_{n}\right) \alpha_{n}+\gamma_{n} \delta_{n}, n \geq 0$, where $\left\{\gamma_{n}\right\}$ is a sequence in $(0,1)$ and $\left\{\delta_{n}\right\}$ is a sequence in $\square$ such that

(i) $\sum_{i=1}^{\infty} \gamma_{n}=\infty$;

(ii) $\limsup \sup _{n \rightarrow \infty} \delta_{n} \leq 0$ or $\sum_{i=1}^{\infty} \gamma_{n}\left|\delta_{n}\right|<\infty$.

Then $\lim _{n \rightarrow \infty} \alpha_{n}=0$.

Lemma 2: Let $X$ be a smooth real Banach space and let $\mathrm{J}$ be the duality map of $X$. Then there holds the inequality

$\|x+y\|^{2} \leq\|x\|^{2}+2\langle x, J(x+y)\rangle, \quad x, y \in X$.

Lemma 3: Let $\left\{x_{n}\right\}$ and $\left\{y_{n}\right\}$ be bounded sequence in a Banach space $X$ such that (Suzuki, 2005, pp.103-123)

$x_{n+1}=\gamma_{n} x_{n}+\left(1-\gamma_{n}\right) y_{n}, n \geq 0$. Where $\left\{\gamma_{n}\right\}$ is a sequence in $[0,1]$ such that $0<\liminf _{n \rightarrow \infty} \gamma_{n} \leq \limsup _{n \rightarrow \infty} \gamma_{n}<1$, assume

$\underset{n \rightarrow \infty}{\limsup }\left(\left\|y_{n+1}-y_{n}\right\|-\left\|x_{n+1}+x_{n}\right\|\right) \leq 0$.

Then $\lim _{n \rightarrow \infty}\left\|y_{n}-x_{n}\right\|=0$.

\section{Main results}

Theorem: Let $X$ be a uniformly smooth Banach space, $C$ a closed convex subset of $X$ and $T: C \rightarrow C$ a nonexpansive 
mapping such that $\operatorname{Fix}(T) \neq \phi$. For any given sequence $\left\{\alpha_{n}\right\}$ in $[0,1], \lambda \in(0,1)$ and $u, x_{0} \in C$, defined $\left\{x_{n}\right\}$ by the iterative algorithm $x_{n+1}=\alpha_{n}\left(\lambda u+(1-\lambda) x_{n}\right)+\left(1-\alpha_{n}\right) J_{r_{n}} x_{n}$,

(i) $\lim _{n \rightarrow \infty} \alpha_{n}=0 \quad$ (ii) $\sum_{i=0}^{\infty} \alpha_{n}=\infty$. Then $\left\{x_{n}\right\}$ convergens strongly to a zero of $A$.

Proof: We first show that $\left\{x_{n}\right\}$ is bounded. Indeed, take a point $p \in \operatorname{Fix}(T)$ to get, using the nonexpansive of $J_{r}$,

$$
\begin{aligned}
\left\|x_{n+1}-p\right\| \leq & \alpha_{n} \lambda\|u-p\|+\alpha_{n}(1-\lambda)\left\|x_{n}-p\right\|+\left(1-\alpha_{n}\right)\left\|J_{r} x_{n}-p\right\| \\
& \leq \alpha_{n} \lambda\|u-p\|+\alpha_{n}(1-\lambda)\left\|x_{n}-p\right\|+\left(1-\alpha_{n}\right)\left\|x_{n}-p\right\| \\
& \leq \alpha_{n} \lambda\|u-p\|+\left(1-\alpha_{n} \lambda\right)\left\|x_{n}-p\right\|
\end{aligned}
$$

So we have for all $n \geq 0,\left\|x_{n}-p\right\| \leq \max \left\{\|u-p\|,\left\|x_{0}-p\right\|\right\}$,

So $\left\{x_{n}\right\}$ is bounded, and also $\left\{J_{r_{n}} x_{n}\right\}$. We have

$\left\|x_{n+1}-J_{r_{n}} x_{n}\right\|=\alpha_{n}\left\|\lambda u+(1-\lambda) x_{n}-J_{r_{n}} x_{n}\right\| \rightarrow 0(n \rightarrow \infty)$

From (2.1) we have $x_{n+1}=\alpha_{n}(1-\lambda) x_{n}+\alpha_{n} \lambda u+\left(1-\alpha_{n}\right) J_{r} x_{n}$

$$
=\alpha_{n}(1-\lambda) x_{n}+\left[1-\alpha_{n}(1-\lambda)\right] \frac{\alpha_{n} \lambda u+\left(1-\alpha_{n}\right) J_{r} x_{n}}{1-\alpha_{n}(1-\lambda)}
$$

We set $\gamma_{n}=1-\alpha_{n}(1-\lambda)$ and $y_{n}=\frac{\alpha_{n} \lambda}{\gamma_{n}} u+\frac{1-\alpha_{n}}{\gamma_{n}} J_{r} x_{n}$

Then we get $x_{n+1}=\left(1-\gamma_{n}\right) x_{n}+\gamma_{n} y_{n}$

It easily to get $\left\{y_{n}\right\}$ (since $\alpha_{n} \rightarrow 0, \gamma_{n} \rightarrow 1$ and $\left\{x_{n}\right\}$ is bounded).

$y_{n+1}-y_{n}=\left(\frac{\alpha_{n+1}}{\gamma_{n+1}}-\frac{\alpha_{n}}{\gamma_{n}}\right) \lambda u+\frac{1-\alpha_{n+1}}{\gamma_{n+1}}\left(J_{r} x_{n+1}-J_{r} x_{n}\right)+\left(\frac{1-\alpha_{n+1}}{\gamma_{n+1}}-\frac{1-\alpha_{n}}{\gamma_{n}}\right) J_{r} x_{n}$

Since $J_{r}$ is nonexpansive and $\frac{1-\alpha_{n+1}}{\gamma_{n+1}}<1$, we get

$\left\|y_{n+1}-y_{n}\right\|-\left\|x_{n+1}-x_{n}\right\| \leq\left(\frac{\alpha_{n+1}}{\gamma_{n+1}}-\frac{\alpha_{n}}{\gamma_{n}}\right) \lambda\|u\|+\left(\frac{1-\alpha_{n+1}}{\gamma_{n+1}}-\frac{1-\alpha_{n}}{\gamma_{n}}\right)\left\|J_{r} x_{n}\right\|$

Since $\alpha_{n} \rightarrow 0, \gamma_{n} \rightarrow 1$, so $\limsup _{n \rightarrow \infty}\left\|y_{n+1}-y_{n}\right\|-\left\|x_{n+1}-x_{n}\right\| \leq 0$

Applying Lemma 3, we have $\lim _{n \rightarrow \infty}\left\|y_{n}-x_{n}\right\|=0$,

since $\alpha_{n} \rightarrow 0$, we get $\lim _{n \rightarrow \infty}\left\|y_{n}-J_{r} x_{n}\right\|=\lim _{n \rightarrow \infty} \frac{\alpha_{n} \lambda}{\gamma_{n}}\left\|u-J_{r} x_{n}\right\|=0$

from (2.5) and (2.6) we get $\lim _{n \rightarrow \infty}\left\|x_{n}-J_{r} x_{n}\right\|=0$

since $z_{t}$ is the unique solution to the equation (1.1), we can write

$$
z_{t}-x_{n}=(1-t)\left(J_{r} z_{t}-x_{n}\right)+t\left(u-x_{n}\right)
$$


Apply lemma 2 to get

$$
\begin{aligned}
\left\|z_{t}-x_{n}\right\|^{2} \leq & (1-t)^{2}\left\|J_{r} z_{t}-x_{n}\right\|^{2}+2 t\left\langle u-z_{n}, J\left(z_{t}-x_{n}\right)\right\rangle \\
\leq & (1-t)^{2}\left\|J_{r} z_{t}-x_{n}\right\|^{2}+\left\|J_{r} x_{n}-x_{n}\right\|\left(2\left\|z_{t}-x_{n}\right\|+\left\|J_{r} x_{n}-x_{n}\right\|\right) \\
& +2\left\langle u-z_{t}, J\left(z_{t}-x_{n}\right)\right\rangle+2 t\left\|z_{t}-x_{n}\right\|^{2}
\end{aligned}
$$

Since $\left\{z_{t}\right\},\left\{x_{n}\right\}$ are bounded, there exists a constant $M>0$,

$$
\left\langle u-z_{t}, J\left(x_{n}-z_{t}\right)\right\rangle \leq\left(t+\frac{\left\|J_{r} x_{n}-x_{n}\right\|}{t}\right) M
$$

So we get $\limsup \left\langle u-z_{t}, J\left(x_{n}-z_{t}\right)\right\rangle \leq t M$

Put

$$
p=Q(u)=s-\lim _{t \rightarrow 0} z_{t}
$$

Since $J$ is uniformly continuous on bounded sets of $X$, we find that

$$
\left\|J\left(x_{n}-z_{t}\right)-J\left(x_{n}-p\right)\right\| \leq \varepsilon_{t} \text {, uniformly on } n .
$$

Where $\quad \varepsilon_{t}>0$ and $\lim _{t \rightarrow 0} \varepsilon_{t}=0$, Let $\beta>0$ satisfy $\left\|u-z_{t}\right\| \leq \beta$ and $\left\|z_{t}-p\right\| \leq \beta$

For all $t \in(0,1)$ and $n$. We have

$$
\begin{gathered}
\left\langle u-p, J\left(x_{n}-p\right)\right\rangle=\left\langle u-z_{t}, J\left(x_{n}-z_{t}\right)\right\rangle+\left\langle u-z_{t}, J\left(x_{n}-p\right)-J\left(x_{n}-z_{t}\right)\right\rangle+\left\langle z_{t}-p, J\left(x_{n}-p\right)\right\rangle \\
\leq\left\langle u-z_{t}, J\left(x_{n}-p\right)\right\rangle+\beta\left(\varepsilon_{t}+\left\|z_{t}-p\right\|\right)
\end{gathered}
$$

It follows (2.10) that, for all $t \in(0,1)$,

$$
\limsup _{n \rightarrow \infty}\left\langle u-p, J\left(x_{n}-p\right)\right\rangle \leq t M+\beta\left(\varepsilon_{t}+\left\|z_{t}-p\right\|\right)
$$

Let $t \rightarrow 0$, we can get $\limsup _{n \rightarrow \infty}\left\langle u-p, J\left(x_{n}-p\right)\right\rangle \leq 0$

Finally, we claim that $x_{n} \rightarrow p$ in norm. Apply Lemma 2 to get

$$
\begin{aligned}
\left\|x_{n+1}-p\right\|^{2} & =\left\|\alpha_{n}(1-\lambda)\left(x_{n}-p\right)+\left(1-\alpha_{n}\right)\left(J_{r} x_{n}-p\right)+\alpha_{n} \lambda(u-p)\right\|^{2} \\
\leq & \left\|\alpha_{n}(1-\lambda)\left(x_{n}-p\right)+\left(1-\alpha_{n}\right)\left(J_{r} x_{n}-p\right)\right\|^{2}+2 \alpha_{n} \lambda\left\langle u-p, J\left(x_{n+1}-p\right)\right\rangle \\
& \leq\left(1-\alpha_{n}\right) \lambda\left\|x_{n}-p\right\|^{2}+2 \alpha_{n} \lambda\left\langle u-p, J\left(x_{n+1}-p\right)\right\rangle
\end{aligned}
$$

Applying lemma 1, and from (2.12) and (2.13) we conclude that $\left\|x_{n}-p\right\| \rightarrow 0$.

So the proof is completed.

References

Kim, Tae-Hwa and Xu, Hongkun. (2005). Strong convergence of modified Mann iterations, Nonlinear Analysis, 61, pp.51-60.

Xu, H. K. (2002). Interactive algorithms for nonlinear operators. Math. Soc. 66. pp.240-256.

Suzuki, T. (2005). Strong convergence theorem for infinite families of no expansive mappings in general Banach spaces. Fixed Point Theory Appl. No. 1. pp.103-123. 